\title{
The Companion to the Central Star of Abell 35
}

\author{
A. A. Gatti ${ }^{1}$, J. E. Drew ${ }^{1}$, S. Lumsden ${ }^{2}$, T. Marsh $^{3}$, C. Moran ${ }^{3}$ and P. Stetson ${ }^{4}$ \\ ${ }^{1}$ Physics Dept., Imperial College of Science Technology and Medicine, London SW7 2BZ, UK; \\ ${ }^{2}$ Anglo-Australian Observatory, PO BOX 296, Epping, NSW 2121, Australia; \\ ${ }^{3}$ Department of Physics, Southampton University, Southampton S09 5NH, UK; \\ ${ }^{4}$ Dominion Astrophysical Observatory, 5071 West Sacnich Road, Victoria, BC V8X 4M6, Canada
}

Close binary systems may undergo the "Common Envelope" (CE) phase when the primary star expands on the red giant branch or the asymptotic giant branch. Filling its Roche Lobe, the primary transfers mass to the companion driving it out of thermal equilibrium and causing it to expand as well. The giant core and the companion star become surrounded by a CE. When sufficient energy is deposited in the circumstellar material this will be ejected and the binary orbit will shrink further (see review by Iben 1995). Planetary nebulae $(\mathrm{PNe})$ with short-period binary nuclei are considered the most probable postCE candidates. Abell 35, Lotr 1 and Lotr 5 (the Abell 35-like objects) are the only three PNe with binary nuclei known to contain a very hot UV-bright primary and a chromospherically active, rapidly rotating, G-K companion that dominates the optical spectrum. The origin of these unusual systems is unclear and hence presents a challenge to theories of binary star evolution. Identified in 1966 by Abell, Abell 35 is possibly the largest PN known ( $\mathrm{D}=1.6 \mathrm{pc}$ at a distance of $360 \mathrm{pc}$, Jacoby 1981) and also the oldest (the kinematical age is 185.000 years from the small expansion velocity of $4.2 \mathrm{~km} / \mathrm{s}$, Bohuski 1974). The bright giant star BD $-22^{\circ} 3467\left(\mathrm{~m}_{\mathrm{v}}=9.6 \mathrm{mag}\right)$ lies off-center within the nebula. A white dwarf was detected at the same location in 1988 in IUE spectra obtained by Grewing and Bianchi. BD $-22^{\circ} 3467$ has a vsin i of $90 \mathrm{~km} / \mathrm{s}$ (Vilhu et al. 1991), variable $\mathrm{H} \alpha$ and $\mathrm{Ca}$ II emission lines associated with chromospheric activity, and a variable light curve ( $\mathrm{P}=0.76$ days, Jasniewicz and Acker 1988 ) probably produced by the rotation of the giant star. All attempts to determine the orbital period have failed, raising doubts as to whether the nucleus of Abell 35 is a close binary at all. In pursuit of this point, we have started a radial velocity study of the giant companion.

We have compiled four epochs of spectroscopic data thus far. The first set was taken in the blue part of the spectrum at intermediate dispersion on 19/02-04/03 1983 with the Las Campanas Du Pont $2.5 \mathrm{~m}$ telescope. At the AAO, echelle spectra $(\Delta \lambda \sim 0.2 \AA)$ were taken on 15-17/04/1992, and grating spectra obtained on $02 / 03 / 1996(\Delta \lambda \sim 0.8 \AA)$ and on $09 / 05 / 1996(\Delta \lambda \sim 1.3 \AA)$, all in the green part of the spectrum. To detect radial velocity variations of $\mathrm{BD}-22^{\circ} 3467$ in the short (few months) or long (few years) timescales due to orbital motion, the heliocentric radial velocity was independently calculated for each night's data. In all cases this was obtained by cross-correlation with radial velocity standards observed on the same night and with the same setup. The radial velocity estimates obtained are listed in table 1. We have also revised the estimate made by Vilhu et al. (1991) from their ESO data of 1987 to be $-30 \pm 9 \mathrm{~km} / \mathrm{s}$. Further, by cross-correlating each spectrum taken in 1992, we have found the short-term variation to be $\sim 0.8 \pm 2.0 \mathrm{~km} / \mathrm{s}$. 
Table 1: Radial velocity estimates of $\mathrm{BD}-22^{\circ} 3467$

\begin{tabular}{|c|c|c|}
\hline observers & date & $\mathbf{v ~ ( k m / s )}$ \\
\hline \hline Stetson & $19 / 02-04 / 031983$ & $-39.9 \pm 3.9$ \\
\hline Drew & $25-27 / 04 / 1992$ & $-40 \pm 2$ \\
\hline Marsh, Moran & $02 / 03 / 1996$ & $-39 \pm 3$ \\
\hline Lumsden & $09 / 05 / 1996$ & $-40 \pm 3$ \\
\hline
\end{tabular}

Thus, within our errors, we observe no radial velocity variations that are plausibly due to orbital motion, either on the short or the long timescale. We find that our collected data for $\mathrm{BD}-22^{\circ} 3467$ are consistent with the radial velocity having remained constant at $-39.6 \pm 0.8 \mathrm{~km} / \mathrm{s}$ (excluding the poorer quality ESO point: when this is included we obtain $-37.7 \pm 2.8 \mathrm{~km} / \mathrm{s}$ ). So, if radial velocity variations of the giant companion exist their amplitude is small (probably $\leq \pm 2 \mathrm{~km} / \mathrm{s}$ ).

Applying Kepler's laws and assuming a number of plausible mass ratios, inclinations and orbital periods we have investigated the available parameter space for the central-star binary. We find that it is most likely that this system has an orbital period of 100-300 years and an intermediate inclination $\left(\sim 45^{\circ}-70^{\circ}\right)$. Lower inclinations are improbable as they would imply a rotational velocity of the companion comparable to its break-up velocity (for $0.7 \mathrm{M}_{\odot} \leq \mathrm{M}_{\text {giant }} \leq 4 \mathrm{M}_{\odot}$ we find $14^{\circ} \leq \mathrm{i}_{\text {break }} \leq 26^{\circ}$ ), whereas higher inclinations produce radial velocity semi-amplitudes too large to be consistent with the limit of \pm 2 $\mathrm{km} / \mathrm{s}$ we have derived for the giant companion. Similarly, periods much longer than 300 years would result in semi-major axis so large that the system should already have been resolved by the IUE observations (assuming a distance of $\sim 300 \mathrm{pc}$ ).

We have also obtained blue spectra of the giant companion ( $\lambda \lambda \mathrm{s} 3900-4600, \Delta \lambda 1.4 \AA$, taken on 09/05/96). By comparing these with 7 spectral type standards observed at the same time spanning spectral classes G6 IV-K0 V, we have confirmed Jacoby's (1981) primarily photometric spectral classification of the star as a G8 III-IV. The hydrogen to metal ratios were not used as composition anomalies could be present. The luminosity classification procedure was then repeated using $\mathrm{Sr}$, an element which could be enriched following mass accretion from an evolved primary. This also pointed to a luminosity class IV, indicating that $\mathrm{Sr}$ does not show evidence of enhancement.

\section{REFERENCES}

Abell, G. O., ApJ., 144, 259 (1966)

Bohuski, T. J. and Smith, M. G., ApJ., 193: 197-203 (1974)

Grewing, M., Bianchi, L., ESA SP-281, Vol 2, p. 177 (1988)

Iben, I., Physics Reports, 2509, 1-94 (1995)

Jacoby, G. H., ApJ., 244, 903-911 (1981)

Jasniewicz, G. and Acker, A., A\&A, 189, L7-L9 (1988)

Vilhu, O., Gustafsson, B., Walter, F. M., A\&A, 241, 167-175, (1991) 